\title{
Oral Mucositis Induced by Methotrexate: A Case Report
}

\section{MAYA VERMA}

The prescription of Methotrexate is well-recognized for various neoplastic diseases. For Rheumatoid arthritis, it is prescribed once a week, low-dose regimen for the treatment of psoriasis and rheumatoid arthritis. Apart from providing therapeutic benefit, it is not devoid of side effects like oral ulceration which may be due to over dosage regarding its once-weekly regime. This case report describes a patient with inherent Methotrexate toxicity that developed oral mucositis due to Methotrexate being given for rheumatoid arthritis.

KEYWORDS: Methotrexate, Rheumatoid Arthritis, Overdose, Toxicity

\section{INTRODUCTION}

Since its introduction in 1950 as a systemic immunosuppressant (dihydrofolate reductase (DHFR) inhibitor), methotrexate (MTX) has been widely used In treatment of various malignancies, early ectopic pregnancy or chronic inflammatory diseases such as some types of carcinoma, rheumatoid arthritis, psoriasis etc. ${ }^{1-3}$ High doses (HD) of MTX (0.5 - 12 g/m2) has shown antiproliferative activity and is used in treatment of cancer while in low doses, (LD) MTX (10 $-40 \mathrm{mg} / \mathrm{mz}$ ) has anti-inflammatory and immunosuppressive properties while Its long-term use is associated with hepatic and pulmonary toxicity. 4,5

This case report discusses the Presence of MTX related mucositis in a 69 year old gentleman and its subsequent treatment leading to complete resolution of the lesion.

\section{CASE REPORT}

A 69 year old gentleman visited a dental camp complaining of oral ulcers (non-recurrent in nature) and dysphagia, who was then referred to a dental clinic for proper examination. Medical history of the patient revealed that the patient was suffering from rheumatoid arthritis, hypertension type II, hypothyroidism and chronic obstructive airway disease. The medications included tablets Doxofylline $400 \mathrm{mg}$ BD, Pantoprazole 40 mg OD, Amlodipine 2.5 mg OD, Nebivolol $2.5 \mathrm{mg}$ OD, T. Atorvastatin $20 \mathrm{mg}$ HS, Clopidogrel 75 mg OD, Levothyroxin 100 mcg OD, Methotrexate $7.5 \mathrm{mg} \mathrm{HS}$, and Methylprednisolone 8 mg OD.
Upon further examination, it was revealed that the patient was prescribed once weekly dose of

Methotrexate $7.5 \mathrm{mg}$ for his long standing Rheumatoid Arthritis, but instead took MTX once daily. No medication error or prescription error was seen, and rather the daily intake of methotrexate was due to an error on the part of the patient itself. As per Ethical considerations, the patient although permitted the findings to be published, he, however declined to publish his pictures, even though the confidentiality of his identity was assured.

Intra-oral clinical examination revealed throat pain, dysphagia and non-recurrent oral ulcer. The lips were fissured and painfully swollen, erosive ulcers in floor of the mouth, buccal and labial mucosa also, the patient complained of generalized weakness and fatigue. The mucositis was revealed to be grade IV. The patient was found to be underweight and malnourished.

The clinical picture gave the impression was of oral mucositis secondary to methotrexate overdose. Treatment was initiated with Inj. Leucovirin Calcium $40 \mathrm{mg}$ every six hourly for 3 days, Folic acid (Tablet) 5 $\mathrm{mg}$ daily for a week along with intravenous fluids. Topical therapy with Choline Salicylate oral gel was initiated, Candid mouth wash and Sodium Bicarbonate mouth was prescribed along with $\mathrm{T}$. Fluconazole, Inj. Mecobalamine. Over time, the patient's lesions healed with regression of all lesions 
within few days after starting local and systemic therapy. The patient was advised on the dosing pattern of MTX and has now been taking MTX on a weekly basis with good treatment compliance.

\section{DISCUSSION}

The present case documented toxicity to MTX in the form of oral mucositis. As MTX can cause many side effects some of which are life threatening, it is of prime importance to recognize them as many of these side effects can be avoided by a close monitoring and adopting good preventive measures. ${ }^{6}$

Often, oral mucositis can become a dose-limiting toxicity and requires the use of opioids for pain relief, It also leads to an increased risk of infections, and has the potential to cause delays in chemotherapy. ${ }^{7}$ Mucositis after MTX is mainly caused by cellular damage to rapidly dividing epithelial cells present along the entire gastrointestinal tract and it is of importance to note that inadequate or delayed leucovorin rescue can lead to impaired epithelial cell growth and regeneration of the affected cells. ${ }^{8,9,10}$

The patient, was suffering from Grade IV mucositis, which is considered to be an oncologic emergency associated with infections, need for parenteral nutrition, increased use of health care resources and finances, and even death. ${ }^{8}$ Therefore the need for an increased screening for oral signs and symptoms is proposed for patients undergoing systemic chemotherapy. Fortunately, the patient described in the present case report did fully recover from the methotrexate-induced mucositis and did not complaint of any oral complications during the last follow up.

\section{REFERENCES}

1. Saigal S, Singh RK, Poddar B. Acute methotrexate toxicity presenting as multiorgan failure and acute pneumonitis: A rare case report. Indian J Crit Care Med. 2012; 16(4): 225-7.

2. Weidmann A, Foulkes AC, Kirkham N, Reynolds N. Methotrexate toxicity during treatment of chronic plaque psoriasis: a case report and review of the literature. Dermatology and therapy. 2014;4(2):145-56. 3. Schmiegelow K. Advances in individual prediction of methotrexate toxicity: A review. Br J Haematol. 2009;46:489-503.

4. Drug Information for the Health Care Professional. $24^{\text {th }}$ ed. Thomson Micromedex; 200:pp1911.

5. Tan KW, Tay YK. A case of acute methotrexate toxicity. Ann Acad Med Singapore. 2011;40:97-9.

6. Gaies E, Jebabli N, Trabelsi S, Salouage I, Charfi R, et al. Methotrexate Side Effects: Review Article. J Drug Metab Toxicol 2012;3:125.

7. Howarda SC, McCormickb J, Puic CH, Buddingtona RK, Harveyd RD. Preventing and Managing Toxicities of High-Dose Methotrexate. The Oncologist 2016;21(12):1471-82.

8. Sonis ST. The pathobiology of mucositis. Nat Rev Cancer 2004;4:277-284.

9. Sonis ST. Mucositis as a biological process: A new hypothesis for the development of chemotherapyinduced stomatotoxicity. Oral Oncol 1998;34:39-43. 10. Sharma K. Managing Methotrexate Toxicity-A Case Report. Int Healthcare Res J 2017;1(1);28-32.
Source of support: Nil, Conflict of interest: None declared
Cite this article as:

Verma M. Oral Mucositis Induced by Methotrexate: A Case Report. Int Healthc Res J. 2018;2(8):195-196. doi: 10.26440/ihrj.v2i8.170

AUTHOR AFFILIATIONS:

1. MBBS, Private Practitioner, Baghpat, India

Corresponding Author:

Dr. Maya Verma

\# 554, Ambedkar Lane

Baghpat, Uttar Pradesh
For article enquiry/author contact details, e-mail at: manuscriptenquiry.ihri@amail.com 